\title{
Fluid-solid coupling characteristics analysis for tripod sliding universal joints
}

\author{
Fuqin Yang ${ }^{*}$, Xin Liu, Shun Zhao, and Linlin Sun \\ College of Mechanical and Electrical Engineering, Qingdao University of Science and Technology, Qingdao, Shandong, PR China
}

Received: 23 August 2018 / Accepted: 6 January 2019

\begin{abstract}
Fluid-solid coupling theory is introduced into tripod sliding universal joint design. The geometric models of the tripod sliding universal joint and lubricant film are established respectively and meshed. Fluid-solid coupling analyses of tripod sliding universal joints in unidirectional and bidirectional scenarios are performed to investigate sleeve and lubricant film coupling as well as sliding pin and lubricant film coupling under various differential pressures and frequencies. In the condition of unidirectional and bidirectional coupling, sleeve and sliding pin deformation and stress increase gradually with differential pressure and frequency. Under various differential pressures and frequencies, tripod sliding universal joint bidirectional coupling deformation and stress are greater than those of unidirectional coupling. The theory of fluid-solid coupling is combined with the tripod sliding universal joint for the first time in this paper. The fluid-solid coupling analysis results are of great significance and benefit to the design, application and marketing of tripod sliding universal joints.
\end{abstract}

Keywords: Tripod sliding universal joint / hydrodynamic oil film / fluid-solid coupling / dynamic mesh technology

\section{Introduction}

Fluid-solid coupling is a cross-disciplinary science that combines higher mathematics, fluid mechanics and solid mechanics to investigate fluid-solid coupling. Research and applications of fluid-solid coupling date to the 19 th century. Tziritas et al. employed the finitedifference method and Gauss-Seidel iterative method to solve the modified transient Reynolds equation [1]. Wodtke et al. employed the fluid-solid coupling method to analyze the thermal elastohydrodynamic lubrication of bearings and gears [2]. Tian et al. created a new rigidflexible multi-system model and employed a fluid-solid coupling method to calculate lubricant film pressure distribution [3]. Liu et al. performed numerical analysis for water turbines via fluid-solid coupling [4]. Xu investigated the bidirectional fluid-solid coupling dynamics modeling of aqueduct structure [5]. Zhang et al. solved fluid-solid thermal coupling system (bearing, lubricating oil and axial diameter) for turbo-generator journal bearings [6]. Mao et al. investigated and analyzed the dynamic characteristics of non-linear oil films of sliding bearings based on fluid-solid coupling [7]. Shi et al. performed fluid-solid coupling simulation analyses for hydrodynamic journal bearings [8]. Yang et al.

\footnotetext{
* e-mail: y_f_q@163.com
}

analyzed the dynamics characteristics of steam turbine bearings based on fluid-solid coupling [9]. These studies provide ideas and methods for the fluid-solid coupling analysis of tripod sliding universal joints.

The fluid-solid coupling analysis of the tripod sliding universal joint is carried out, which makes the analysis results closer to the reality. However, due to the complexity of fluid-solid coupling system, the long calculation period and the difficulty of calculation, previous study on lubrication characteristics of the joints did not take into account the deformation behavior of solids under the action of flow field and the reaction of the position and shape of solids to flow field [10,11]. In this paper, fluid-solid coupling analyses of sleeve and lubricant film coupling as well as sliding pin and lubricant film coupling in unidirectional and bidirectional scenarios are performed under different working conditions. The two coupling results are compared and analyzed.

\section{Fluid domain analysis}

\subsection{Fluid control equation}

Compared with the normal Reynolds equation, the fluid dynamics control equation simulates the lubricating characteristics of tripod sliding universal joints more accurately. 


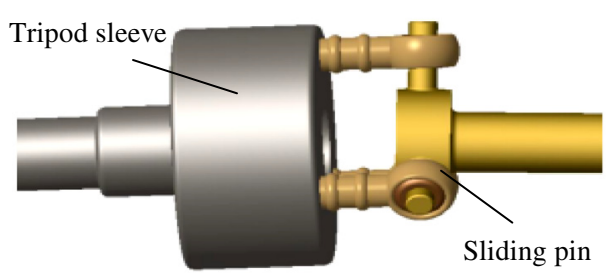

(a) Tripod sliding universal joint

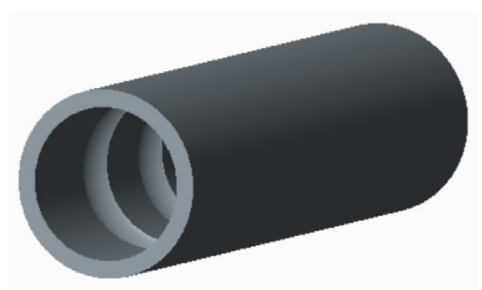

(b) Lubricant film

Fig. 1. Three-dimensional diagram of tripod sliding universal joint and lubricant film.

1. Mass conservation equation

According to mass conservation, control body internal mass increment equals the mass flowing into the control body:

$$
\frac{D \rho}{D t}+\rho \vec{\nabla} \cdot \overrightarrow{\boldsymbol{v}}=0
$$

In formula (1), $\frac{D}{D t}$ is the particle derivative, $\rho$ is the fluid density, $\overrightarrow{\boldsymbol{v}}$ is the velocity vector and $\vec{\nabla}$ is the gradient vector.

\section{Momentum conservation equation}

According to the conservation of momentum, the control body momentum increment equals the sum of influent momentum, surface force impulse and volume force impulse. The differential form of fluid motion equation is:

$$
\rho \frac{D \overrightarrow{\boldsymbol{v}}}{D t}-\rho \overrightarrow{\boldsymbol{f}}-\vec{\nabla} \times \overrightarrow{\boldsymbol{\imath}}=\overrightarrow{\mathbf{0}}
$$

In formula (2), $\overrightarrow{\boldsymbol{f}}$ is the body force, and $\overrightarrow{\boldsymbol{\imath}}$ is the surface tension. In the rectangular coordinate system, stress tensor is as follows:

$$
\overrightarrow{\boldsymbol{\imath}}=\left[\begin{array}{ccc}
\boldsymbol{\sigma}_{x} & \boldsymbol{}_{x y} & \boldsymbol{\imath}_{x z} \\
\boldsymbol{l}_{y x} & \boldsymbol{\sigma}_{y} & \boldsymbol{\imath}_{y z} \\
\boldsymbol{t}_{z x} & \boldsymbol{}_{z y} & \boldsymbol{\sigma}_{z}
\end{array}\right]
$$

\section{Energy conservation equation}

Conservation of energy means that the sum of mechanical energy and all internal energy in a system is equal to the total energy of the system. According to the energy conservation equation, control body energy variation equals the sum of influent energy, surface force work, volume force work and influent heat:

$$
\frac{D E}{D t}=\frac{D Q}{D t}+\frac{D W}{D t}
$$

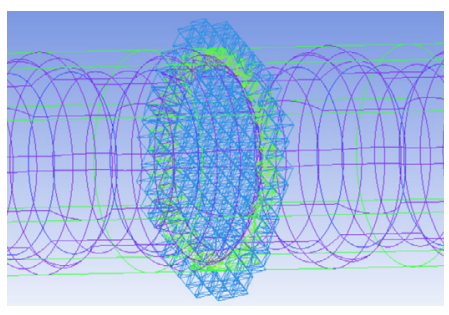

(a) Unstructured mesh

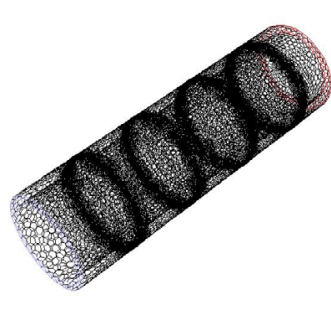

(b) Polyhedron mesh
Fig. 2. Lubricant film mesh.

In formula (3), $E$ is the internal energy of the system, $Q$ is the heat transferred into the system from the external environment and $W$ is the external work on the system.

\subsection{Three-dimensional model and boundary condition}

The Pro/E modeling system is used to build the model of the tripod sliding universal joint and lubricant film [12], as shown in Figure 1.

As the lubricant film between the tripod sleeve and sliding pin in the tripod sliding universal joint is very thin, the ratio between the lubricant film length and thin wall dimension is very large, which makes meshing difficult. Therefore, an unstructured mesh is created using the octree algorithm in ICEM (shown in Fig. 2a), which has superior robustness. Although unstructured mesh facilitates complex area meshing, when the unstructured mesh is imported into FLUENT for solving, the mesh may result in a significant expansion ratio that could affect calculation precision. Therefore, the unstructured mesh should be converted into a polyhedron mesh for calculation, as shown in Figure 2b.

FLUENT and ANSYS WORKBENCH are combined to analyze fluid-solid coupling $[13,14]$. The flow field is set as an incompressible flow field and the SIMPLE velocitypressure algorithm is employed to obtain a solution. The k$\varepsilon$ turbulent flow model is also employed. When solving the tripod sliding universal joint lubrication model, the outlet pressure is equal to atmospheric pressure and P100 lubricating oil is selected as the lubricant. The material attributes of the tripod sleeve and sliding pin are listed in Table 1 . The characteristics of the lubricating oil are listed in Table 2.

\subsection{Flow field analysis results}

\subsubsection{Flow field analysis results under various} differential pressures

Differential pressure is the difference between pressureinlet and pressure-outlet in lubricating area. According to the actual working condition of the joint, flow field distribution data of lubricant films are obtained via FLUENT under various differential pressures $(5 \mathrm{e}+7$, $6.5 \mathrm{e}+7,8 \mathrm{e}+7$ and $1 \mathrm{e}+8 \mathrm{~Pa})$.

Figure 3a shows lubricant film pressure nephogram when the differential pressure is $5 \mathrm{e}+7 \mathrm{~Pa}$. The scatter diagram in Figure $3 \mathrm{~b}$ shows the maximum fluid pressure 
Table 1. Material attributes.

\begin{tabular}{llll}
\hline Tripod sleeve & Parameter & Sliding pin & Parameter \\
\hline Material & $55 \#$ steel & Material & GCr15 \\
Density $\left(\mathrm{kg} / \mathrm{m}^{3}\right)$ & 7850 & Density $\left(\mathrm{kg} / \mathrm{m}^{3}\right)$ & 7850 \\
Elastic modulus $(\mathrm{GPa})$ & 201 & Elastic modulus $(\mathrm{GPa})$ & 205 \\
Poisson ratio & 0.3 & Poisson ratio & 0.29 \\
Thermal expansion coefficient $\left(10^{-6} /{ }^{\circ} \mathrm{C}\right)$ & 11.59 & Thermal expansion coefficient $\left(10^{-6} /{ }^{\circ} \mathrm{C}\right)$ & 10 \\
Specific heat capacity $\left(\mathrm{J} / \mathrm{kg} \cdot{ }^{\circ} \mathrm{C}\right)$ & 468.9 & Specific heat capacity $\left(\mathrm{J} / \mathrm{kg} \cdot{ }^{\circ} \mathrm{C}\right)$ & 495 \\
Thermal conductivity $\left(\mathrm{W} / \mathrm{m} \cdot{ }^{\circ} \mathrm{C}\right)$ & 48.15 & Thermal conductivity $\left(\mathrm{W} / \mathrm{m} \cdot{ }^{\circ} \mathrm{C}\right)$ & 38 \\
\hline
\end{tabular}

Table 2. Characteristics of P100 lubricating oil at $295 \mathrm{~K}$ (temperature).

\begin{tabular}{lll}
\hline$\Phi_{0}(\mathrm{~Pa} \cdot \mathrm{s})$ & $\alpha\left(\mathrm{Pa}^{-1}\right)$ & $\rho_{0}\left(\mathrm{~kg} / \mathrm{m}^{3}\right)$ \\
\hline 0.214 & $2.354 \times 10^{-8}$ & 870 \\
\hline
\end{tabular}

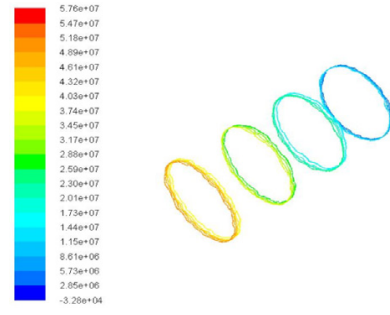

(a) Stress diagram



(b) Scatter diagram
Fig. 3. Lubricant film pressure.

under various differential pressures. Other parts are ignored and only the place with the thinnest lubricant film, i.e., the actual lubricating area of the lubricant film, is observed. Figure 3 shows that the maximum lubricant film pressure is at the inlet and the minimum pressure is at the outlet. Overall lubricant film pressure and the maximum fluid pressure increase with differential pressure. Negative pressure in the lubricant film means the lubricant film could rupture and cavitation could occur. In the pressure nephogram, the lubricant film rupture region has a negative value. However, the actual pressure is 0 , and a rising negative pressure increases gasification. This reduces the mixture density of the lubricating oil, which is unfavorable to lubricant film bearing and affects the lubricating capability of the lubricant film.

\subsubsection{Flow field analysis results under various frequencies}

With constant differential pressure, various frequencies are selected for flow field analysis to obtain lubricant film pressure nephograms and a scatter diagram (Fig. 4).

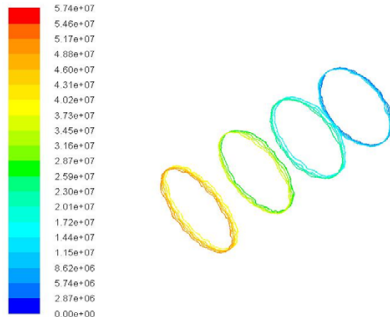

(a) Stress diagram

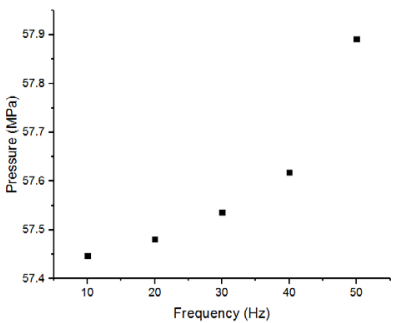

(b) Scatter diagram
Fig. 4. Lubricant film pressure.

Figure 4a shows lubricant film pressure nephogram when the frequency is $10 \mathrm{~Hz}$. The scatter diagram in Figure 4b shows the maximum fluid pressure under various frequencies $(10,20,30,40$ and $50 \mathrm{~Hz})$.

From the analysis results, lubricant film pressure changes with frequency. Under constant differential pressure, lubricant film pressure increases with frequency and the probability of lubricant film rupture also increases. After lubricant film rupture, the rupture region increases with frequency. As shown in Figure 4b, when the frequency is in the range of $10-40 \mathrm{~Hz}$, the amplitude of lubricant film pressure variation is moderate and the difference is insignificant. When frequency increases to $50 \mathrm{~Hz}$, the amplitude of lubricant film pressure variation increases suddenly, and the increment is apparent.

\section{Unidirectional coupling}

\subsection{Fluid-solid coupling results under various differential pressures}

\subsubsection{Tripod sleeve}

A tripod sliding universal joint under fluid-solid coupling is simulated to obtain overall tripod sleeve deformation under various differential pressures. The sleeve deformation nephogram when the differential pressure is $5 \mathrm{e}+7 \mathrm{~Pa}$ and a scatter diagram are shown in Figure 5.

Figure 5 shows that sleeve deformation increases gradually with differential pressure. The maximum tripod sleeve deformation is close to input axis, where the coupling 


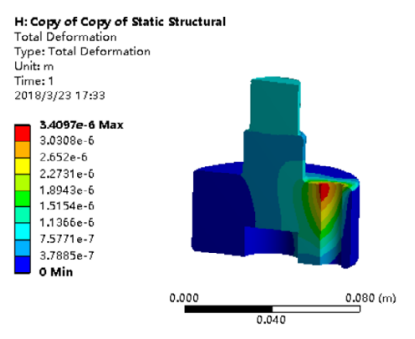

(a) Deformation diagram

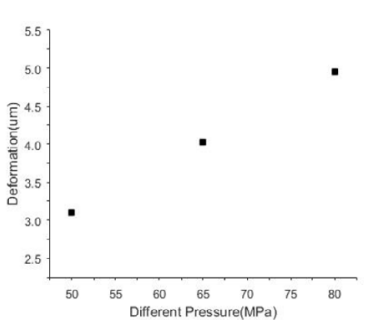

(b) Scatter diagram

Fig. 5. Overall deformation nephogram of sleeve.

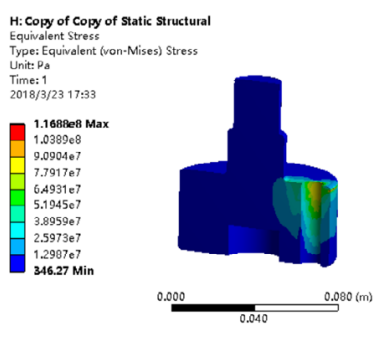

(a) Stress diagram

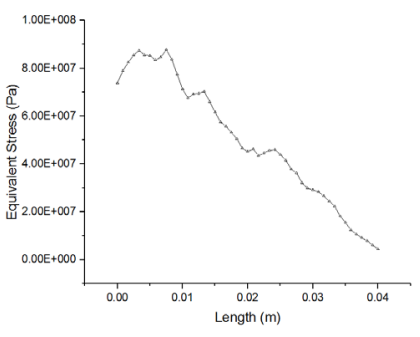

(b) Stress path curve

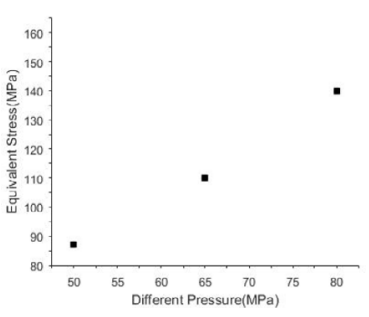

(c) Scatter diagram

Fig. 6. Sleeve stress nephogram.

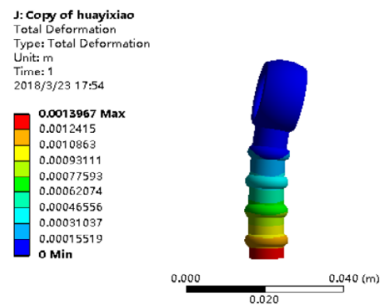

(a) Deformation diagram

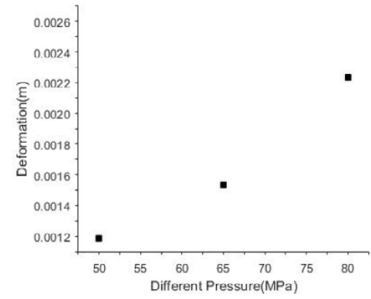

(b) Scatter diagram

Fig. 7. Overall deformation nephogram of tripod sliding pin.

deformation effect of the lubricant film fluid domain and sleeve solid domain is the most apparent. Along the downward direction of the axis, sleeve deformation reduces gradually. Although such deformation is moderate for the sleeve, it is substantial compared with lubricant film thickness.

Figure $6 \mathrm{a}$ is sleeve stress diagram when the differential pressure is $5 \mathrm{e}+7 \mathrm{~Pa}$. A new path is created along the holes in the sleeve from top to bottom and a corresponding stress curve is generated, as shown in Figure 6b. Figure 6b shows that sleeve stress near the first ring bulge of the wearresistant structure close to inlet has a clear fluctuation; then, the fluctuations decline gradually and almost all fluctuations occur at the ring bulge. The maximum stress is at the junction between the first ring bulge and the sleeve, where stress concentration is likely to occur. When stress exceeds an allowed value, it could reduce the service life of the sleeve or cause accidents, such as fracture. The scatter diagram in Figure 6c shows that maximum sleeve stress increases with differential pressure and the variation rate is considerable. This means that differential pressure has a significant impact on sleeve stress.

\subsubsection{Sliding pin}

A tripod sliding universal joint under fluid-solid coupling is simulated to obtain overall tripod sliding pin deformation under various differential pressures. The sliding pin deformation nephogram when the differential pressure is $5 \mathrm{e}+7 \mathrm{~Pa}$ and a scatter diagram are shown in Figure 7 .

Figure 7 shows that the maximum deformation of the sliding pin occurs at the first thinnest lubricant film close to the end. Overall sliding pin deformation along the axis demonstrates the trend of gradual change, which results in local convergence and diffusion clearance and thus changes the structure of the lubricant film at the clearance of the tripod sliding universal joint and affects parameters in the lubricant film flow field. Sliding pin deformation gradually increases with differential pressure and becomes more substantial compared with sleeve deformation. This means that the sliding pin is more susceptible to the flow field, which is related to the sliding pin material and dimensions.

Figure 8a shows slide pin stress diagram under various differential pressures. Figure $8 \mathrm{~b}$ shows a path created along the sliding pin from bottom to top and the corresponding 


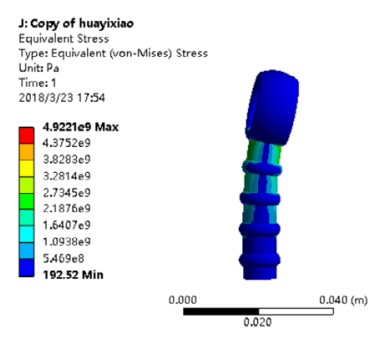

(a) Stress diagram

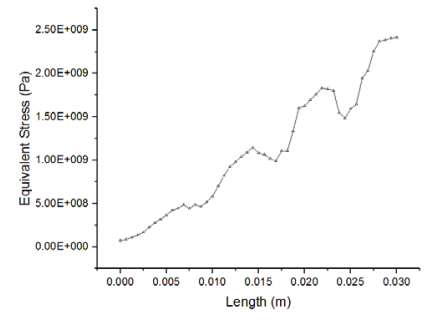

(b) Stress path curve

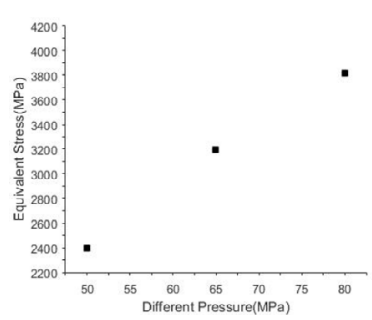

(c) Scatter diagram

Fig. 8. Stress nephogram of sliding pin.

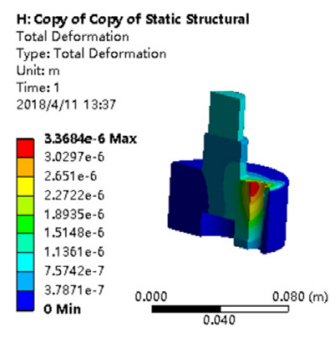

(a) Deformation diagram

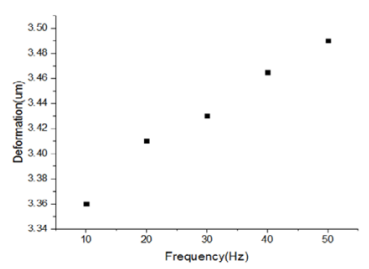

(b) Scatter diagram
Fig. 9. Overall sleeve deformation nephogram.

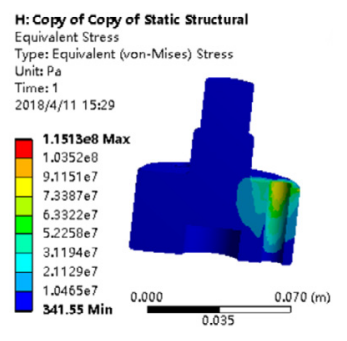

(a) Stress diagram

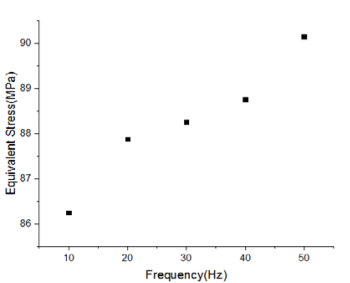

(b) Scatter diagram
Fig. 10. Sleeve stress nephogram.

stress curve. Figure $8 \mathrm{a}$ and b show that sliding pin stress declines gradually from top to bottom. Stress variation is not a smooth curve, but fluctuates. Fluctuating points concentrate at the ring bulge of the wear-resistant structure. There are three changes from top to bottom. The first two have significant stress fluctuations and the last one is relatively smooth. Stress concentrates at the ring bulge of the sliding pin. These areas have the highest levels of stress and strain. Deformation or fracture is likely to occur, which affects the service life of the tripod sliding universal joint. Just like the sleeve, the scatter diagram in Figure 8c shows that maximum sliding pin stress increases with differential pressure and the variation rate is considerable.

\subsection{Fluid-solid coupling results under various frequencies}

\subsubsection{Tripod sleeve}

Figure 9 shows sleeve deformation nephogram when the frequency is $10 \mathrm{~Hz}$ and a scatter diagram. Figure 9 shows that the maximum sleeve deformation is at the inlet and

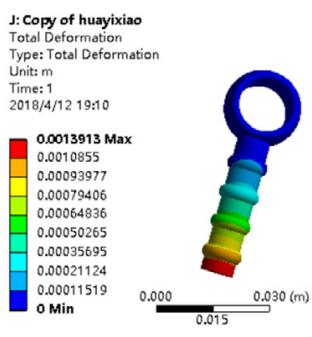

(a) Deformation diagram

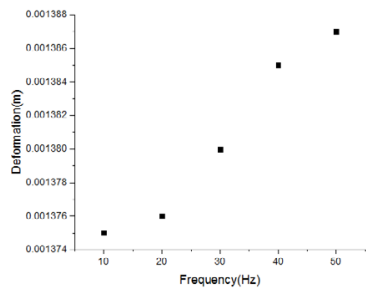

(b) scatter diagram
Fig. 11. Overall deformation nephogram of sliding pin.

then gradually reduces along the direction toward the interior. Sleeve deformation also gradually increases with frequency. When the differential pressure increases, the sleeve deformation changes significantly. However, in contrast to the differential pressure, the frequency variation induced deformation variation is relatively small, and the deformation growth rate is stable.

Figure 10 shows sleeve stress nephogram when the frequency is $10 \mathrm{~Hz}$ and a scatter diagram. As shown in Figure 10, the maximum sleeve stress increases with frequency and the range of the maximum stress gradually expands. In the range of $20-40 \mathrm{~Hz}$, the stress variation rate is moderate and stable; however, at $10 \mathrm{~Hz}$ or $50 \mathrm{~Hz}$, the curve variation rate has an apparent significant change. This means that when frequency is too high or too low, stress change is apparent.

\subsubsection{Sliding pin}

Figure 11 shows sliding pin deformation nephogram when the frequency is $10 \mathrm{~Hz}$ and a scatter diagram. As shown in Figure 11, the overall deformation of the sliding pin in the tripod sliding universal joint gradually increases with frequency; deformation at the ring bulge is greater than that of the sliding bar. When the frequency is low, deformation variation is small. As frequency increases, deformation increases significantly. When the frequency grows to a certain level, the deformation increment slows; however, the overall trend grows. The trend of deformation variation rate initially increases and subsequently decreases.

Figure 12 shows sliding pin stress nephogram when the frequency is $10 \mathrm{~Hz}$ and a scatter diagram. As shown in Figure 12, the sliding pin stress variation trend is similar to 


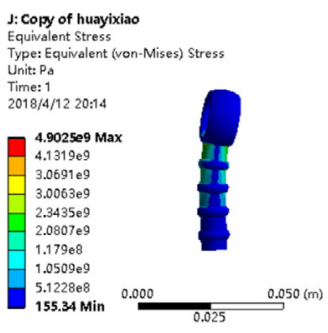

(a) Stress diagram

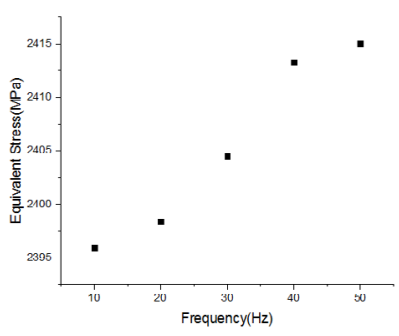

(b) scatter diagram
Fig. 12. Stress nephogram of sliding pin.

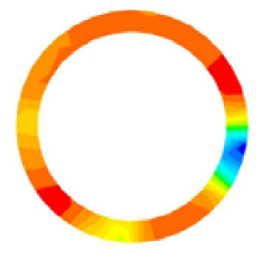

(a)Bidirectional

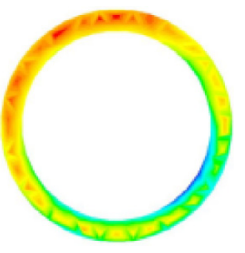

(b)Unidirectional
Fig. 13. Lubricant film pressure.

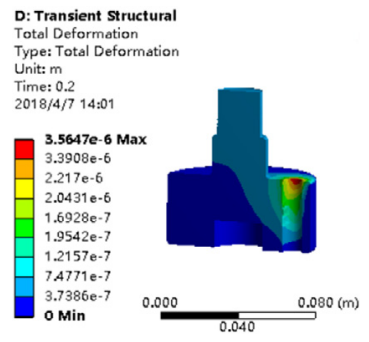

(a) Sleeve deformation

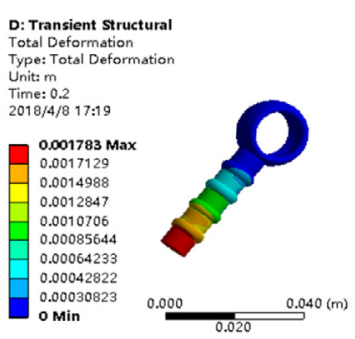

(c) Sliding pin deformation

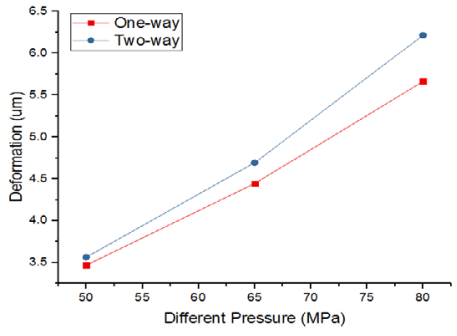

(b) Comparison of sleeve deformations

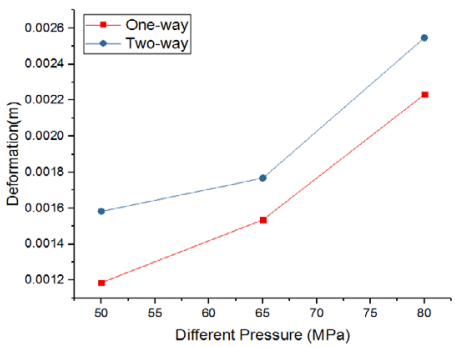

(d) Comparison of sliding pin deformations
Fig. 14. Deformation diagrams under various differential pressures.

the deformation variation trend. Both increase initially and subsequently decrease. Stress also increases with frequency. In the mid-range of frequency, stress variation rate is stable and stress increases quickly. When frequency reaches $50 \mathrm{~Hz}$, the increasing trend slows down significantly. Compared with the tripod sleeve, the sliding pin is more susceptible to equivalent stress. Additionally, higher velocity results in higher fluid dynamic pressure and a higher peak value of equivalent stress. Consequently, the sliding pin exceeds the allowed stress, which results in wear deformation or fracture.

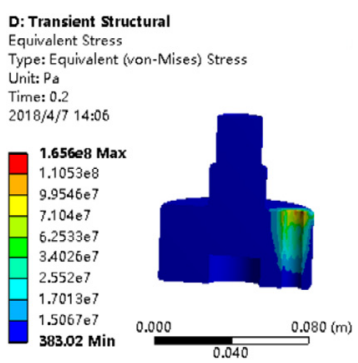

(a) Sleeve stress

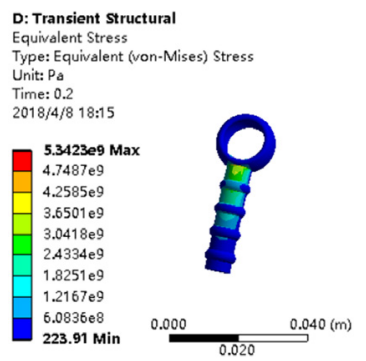

(c) Sliding pin stress

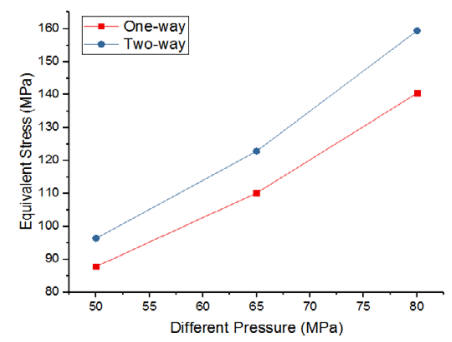

(b) Comparison of sleeve stresses

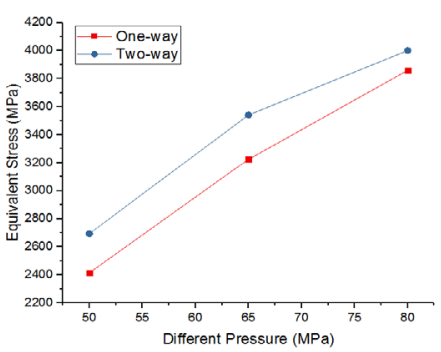

(d) Comparison of sliding pin stresses
Fig. 15. Stress diagrams under various differential pressures.

\section{Bidirectional coupling}

A dynamic mesh is defined by the Profile and UDF macros in FLUENT [15]. Coupling data are configured based on the parameters in Tables 1 and 2. The time step is defined as $0.001 \mathrm{~s}$ and the solution stop time is $0.2 \mathrm{~s}$. The initial loop sequence starts from the flow field calculation [16].

Figure 13 shows a lubricant film pressure diagram. Figure 13 shows that, compared with unidirectional coupling, the high-pressure range in bidirectional coupling expands significantly. This is because, in unidirectional coupling, the fluid domain only transfers pressure to the solid domain and the solid domain cannot feed data back to the fluid. In bidirectional coupling, however, the solid is under flow field pressure and displacement is fed back to the flow field. Based on the mesh node diffusion model, the flow field starts to calculate displacement diffusion, then generates new fluid mesh after deformation.

\subsection{Fluid-solid coupling results under various differential pressures}

Figure 14 shows deformation diagrams under various differential pressures. Figure 15 shows stress diagrams under various differential pressures. When the fluid domain exterior wall and solid domain interior wall are coupled and other conditions are identical, sleeve deformation and stress increase with differential pressure; the results for bidirectional coupling are larger than those for unidirectional coupling. Additionally, higher differential pressure induces a more significant difference between the results in unidirectional coupling and those in bidirectional coupling. Compared with unidirectional coupling analysis, bidirectional coupling analysis is more complex because both convergence and computing cost need to be considered. 


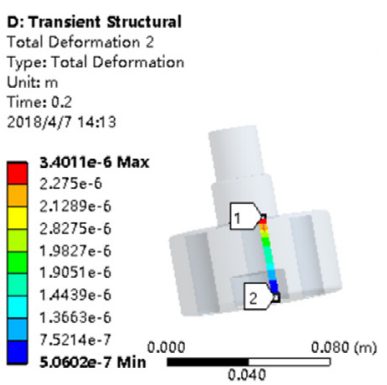

(a) Sleeve deformation

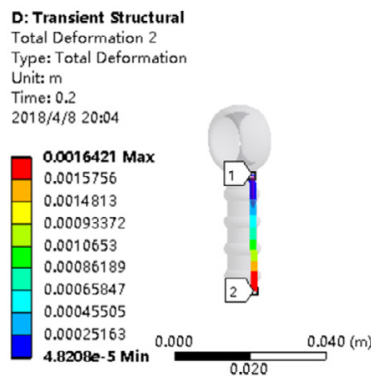

(c) Sliding pin deformation

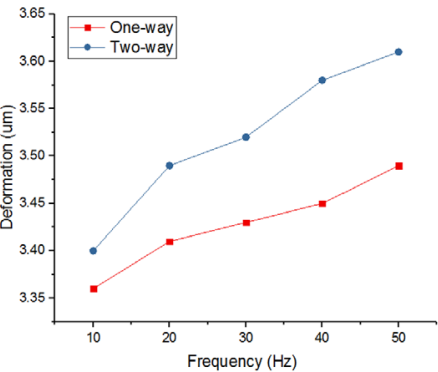

(b) Comparison of sleeve deformations

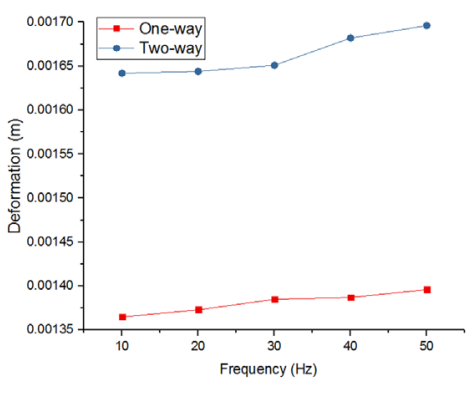

(d) Comparison of sliding pin deformations

Fig. 16. Deformation diagrams under various frequencies.

However, the lubricant film in the tripod sliding universal joint is very thin (less than $1 \mu \mathrm{m}$ ) and extremely thin lubricant film has the following consequence: slight solid deformation results in significant changes in the lubricant film. In terms of numerical precision, bidirectional coupling is superior.

Figures 14 and 15 show that, when the fluid domain interior wall and solid domain exterior wall are coupled and other conditions are identical, sliding pin deformation and stress increase with differential pressure. The line chart shows that bidirectional coupling shows more significant deformation and stress than unidirectional coupling. Compared with the sleeve, the sliding pin has a more significant difference between unidirectional coupling and bidirectional coupling. In general, stronger unidirectional coupling results in a more significant calculation deviation. The comparisons in Figures 7, 8 and Figures 11, 12 show that unidirectional coupling has a more significant impact on the sliding pin, which means sliding pin unidirectional coupling has a larger error.

\subsection{Fluid-solid coupling results under various frequencies}

As shown in Figure 16a and c, a path curve from 1 to 2 is set up to observe the variation of the deformations of the sleeve and the sliding pin on the specified path under various frequencies and to record the data in Figure 16b and d.

The sleeve and sliding pin deformation analysis results are shown in Figure 16. Figure 16 shows that the sleeve has less severe deformation than the sliding pin. When frequency increases, the deformation diagrams of both the sliding pin and the sleeve increase. The sleeve deformation variation rate is more apparent than the sliding pin variation rate. The difference between

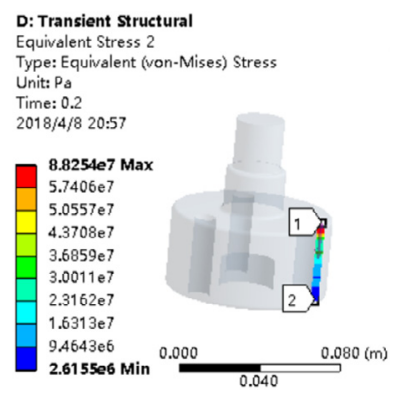

(a) Sleeve stress

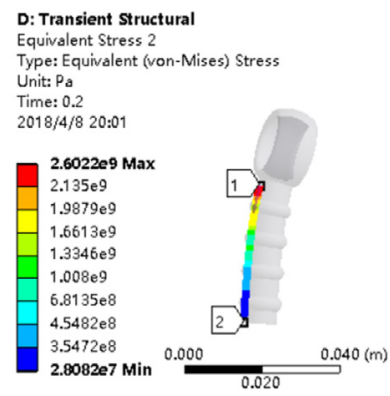

(c) Sliding pin stress

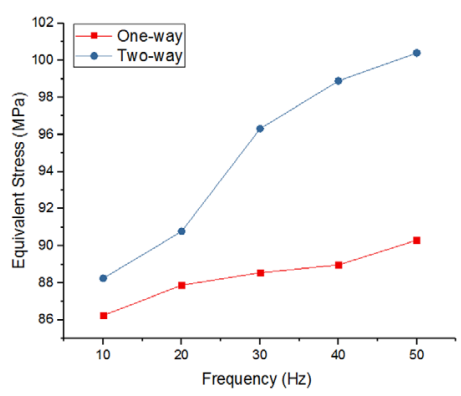

(b) Comparison of sleeve stresses

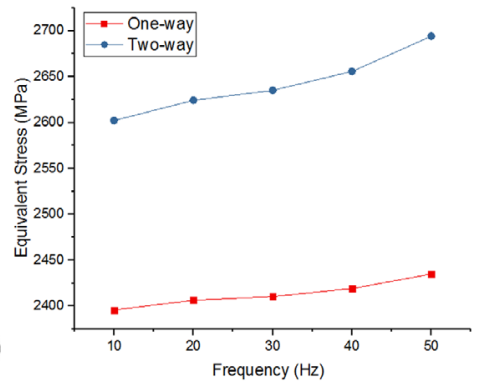

(d) Comparison of sliding pin stresses
Fig. 17. Stress diagrams under various frequencies.

unidirectional coupling and bidirectional coupling is smaller at low frequencies. As frequency rises, the difference between deformation variation rates under unidirectional coupling and bidirectional coupling gradually increase. The difference between the sliding pin deformations under unidirectional coupling and bidirectional coupling is significant; however, the deformation variation rates are relatively gentle and the growth trends are similar. As frequency rises, the bidirectional variation rate is more apparent than the unidirectional variation rate.

As shown in the Figure 17a and c, a path curve from 1 to 2 is set up to observe the variation of the stresses of the sleeve and the sliding pin on the specified path under various frequencies and to record the data in Figure 17b and d.

The sleeve and sliding pin stress analysis results are shown in Figure 17. Similar to the trend of the deformation curve, sleeve and sliding pin stress variations also increase with frequency. For the sliding pin, the difference between the results for unidirectional coupling and those for bidirectional coupling is greater than that for the sleeve. Figure 17 shows that when the frequency is low, the difference in sleeve stress variations between unidirectional and bidirectional coupling is moderate. However, as frequency increases, the sleeve stress variation rate is significantly larger than the deformation variation rate. Higher frequency results in a more significant difference between the unidirectional coupling result and the bidirectional coupling result. The sliding pin stress variation rate is moderate; however, the overall stress difference is significant. Based on the unidirectional coupling analysis in Section 2, despite various differential pressures and frequencies, unidirectional coupling and bidirectional coupling have a more significant impact for the sliding pin; the sliding pin is more susceptible to stress concentration and is likely to develop bending, which affects the service life of the joint. 


\section{Conclusions}

1. Lubricant film pressure increases with differential pressure and frequency.

2. In unidirectional coupling, tripod sleeve and sliding pin deformations and stresses increase with differential pressure and frequency. Sliding pin deformation and stress variations are more apparent.

3. In bidirectional coupling, sleeve and sliding pin deformations and stresses increase with differential pressure and frequency.

4. Under various differential pressures and frequencies, the deformation and stress in bidirectional coupling are greater than those for unidirectional coupling; the differences in sleeve deformations and stresses between unidirectional coupling and bidirectional coupling increase gradually with differential pressure and frequency. Differences in sliding pin deformations and stresses between unidirectional and bidirectional coupling are relatively stable; however, they are significantly larger for the sleeve.

\section{Nomenclature}

$\rho \quad$ Fluid density

$\overrightarrow{\boldsymbol{v}} \quad$ Velocity vector

$\vec{\nabla} \quad$ Gradient vector

$\vec{f} \quad$ Body force

$\overrightarrow{\boldsymbol{\imath}} \quad$ Surface tension

$E \quad$ Internal energy of the system

$Q$ Heat transferred into the system from the external environment

$W$ External work on the system

Acknowledgement. This paper was supported by the key research and development project (Science and technology for public welfare) program of Shandong Province (Grant NO.2018GGX103015).

\section{References}

[1] G. Tziritas, Recursive and iterative estimation of the twodimensional velocity field and reconstruction of threedimensional motion, Signal Process. 16 (1989) 53-72
[2] M. Wodtke, A. Olszewski, M. Wasilczuk, Application of the fluid-structure interaction technique for the analysis of hydrodynamic lubrication problems, Proc. Inst. Mech. Eng. Part J J. Eng. Tribol. 227 (2013) 888-897

[3] Q. Tian, Y. Sun, C. Liu et al., Elastohydrodynamic lubricated cylindrical joints for rigid-flexible multibody dynamics, Comput. Struct. 114-115 (2013) 106-120.

[4] D. Liu, S. Liu, Y. Wu et al., Numerical simulation of hydraulic turbine based on fluid-structure coupling, Chin. J. Hydrodyn. (2008) 345-351.

[5] J.G. Xu, H. Chen, B. Wang et al., Dynamic modeling study of aqueduct structure with fluid-structure coupling, J. Nanjing Univ. Sci. Technol. 2005.

[6] P.L. Zhang, H. Zhang, Z.D. Wu et al., Application of the fluid-solid-heat interaction method to study the lubrication of a 1000-MW turbine generator journal bearing, Large Electr. Mach. Hydraulic Turbine 05 (2013) 30-33.

[7] W.G. Mao, X. Han, G.P. Liu, Research on the dynamic characteristics of nonlinear slide bearing oil films based on fluid-solid interaction, China Mech. Eng. 25 (2014).

[8] Y.X. Shi, Y. Yang, Y.Z. Wang, Simulation analysis of fluidsolid coupling for hydrodynamic sliding bearings, Process Equip. Piping 51 (2014) 43-46

[9] J.G. Yang, T. Cai, M. Gao, Three-dimensional fluid-solid analytical model of steam turbine bearings, Proc. Chin. Soc. Electr. Eng. 24 (2004) 147-151

[10] X.F. Wang, D.G. Chang, J.Z. Wang, Kinematic investigation of tripod sliding universal joint based on coordinate transformation, Multibody Syst. Dyn. 22 (2009) 97-113

[11] Y. Zhou, D.G. Chang, S.M. Li, Analysis on grease lubrication properties of the tripod sliding universal coupling in automotive transmission shaft, Ind. Lubr. Tribol. 69 (2017) 598-604

[12] Y. Zhou, Study on the dual automotive universal joint friction and lubrication, Qingdao University of Science and Technology, 2017.

[13] S. Zhang, L.J. Xiao, Q. Liu et al, Tribology performance testing and fluid-solid-thermal coupling simulation of automotive ventilated disc brakes, Automot. Eng. 39 (2017) 675-682.

[14] L.D. Li, S. Li, Numerical simulation technology based on CFX and Workbench software, China Measur. Test 36 (2010) $79-80+83$.

[15] T. Li, Study on the transient characteristics of hydrodynamic mechanical seal multi-field interaction, Jiangsu University, 2017.

[16] S.J. Zheng, Liquid-solid-thermal coupling analysis and flow visualization of cartridge poppet valves, Taiyuan University of Technology, 2015.

Cite this article as: F. Yang, X. Liu, S. Zhao, L. Sun, Fluid-solid coupling characteristics analysis for tripod sliding universal joints, Mechanics \& Industry 20, 201 (2019) 